\title{
Smoothness in Relative Geometry
}

\author{
Florian Marty \\ fmarty@math.ups-tlse.fr \\ Université Toulouse III - Laboratoire Emile Picard
}

\begin{abstract}
In [TVa, Bertrand Toën and Michel Vaquié defined a scheme theory for a closed monoidal category $(\mathcal{C}, \otimes, 1)$. In this article, we define a notion of smoothness in this relative (and not necessarily additive) context which generalize the notion of smoothness in the category of rings. This generalisation consists practically in changing homological finiteness conditions into homotopical ones using Dold-Kahn correspondence. To do this, we provide the category $s \mathcal{C}$ of simplicial objects in a monoidal category and all the categories $s A-\bmod , s A-\operatorname{alg}(A \in \operatorname{scomm}(\mathcal{C}))$ with compatible model structures using the work of Rezk in $[\mathrm{R}]$. We give then a general notions of smoothness in $s \operatorname{Comm}(\mathrm{C})$. We prove that this notion is a generalisation of the notion of smooth morphism in the category of rings, is stable under compositions and homotopic pushouts and provide some examples of smooth morphisms in $\mathbb{N}-$ alg and $\operatorname{Comm}($ Set $)$.
\end{abstract}

\section{Contents}

\section{Abstract}

\section{Introduction}

\section{Preliminaries}

\section{General Theory}

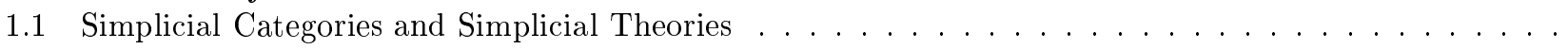

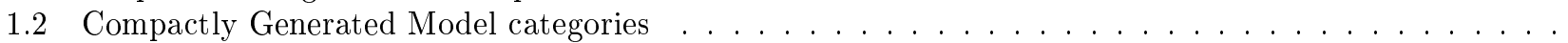

1.3 Categories of Modules and Algebras . . . . . . . . . . . . . . . . . . . . .

1.4 Finiteness Conditions . . . . . . . . . . . . . . . . . . . . . . .

1.5 A Definition for Smoothness . . . . . . . . . . . . . . . . . . .

2 Simplicial Presheaves Cohomology

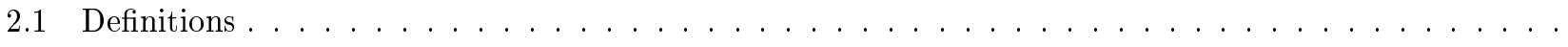

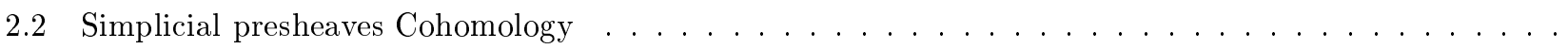

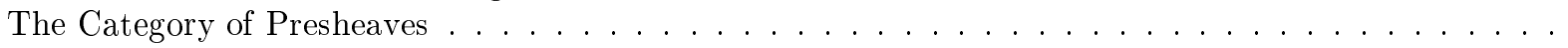

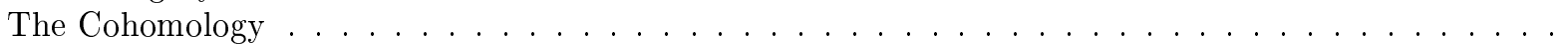

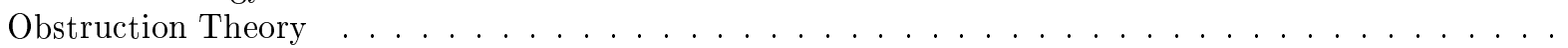

2.3 Simplicial Modules Cohomology . . . . . . . . . . . . . . . . . . . . . . . . . 12

\section{Examples}

3.1 The Category $\left(\mathbb{Z}-\bmod , \otimes_{\mathbb{Z}}, \mathbb{Z}\right) \ldots \ldots \ldots \ldots$

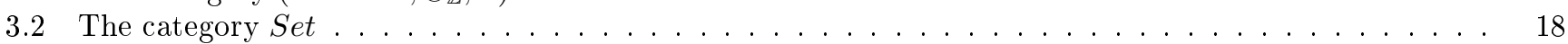

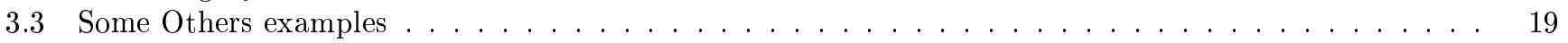

\section{Introduction}

In [TVa], Bertrand Toën and Michel Vaquié defined a scheme theory for a closed monoidal category $(\mathcal{C}, \otimes, 1)$. In this article, we define a notion of smoothness in this relative context which generalize the notion of smoothness in the category of rings. The motivations for this work are that interesting objects in the non additive contexts $\mathcal{C}=E n s$ or $\mathbb{N}-\bmod$ are expected not to be schemes but Stacks. A theorem asserts for $\mathcal{C}=\mathbb{Z}-\bmod$ that quotients of schemes by smooth group schemes are in fact algebraic stacks. A near theorem is expected in a relative context. The first step is 
to get a definition for smooth morphism.

The following theorem give the good definition of smoothness that can be generalised to the relative context:

Theorem 0.1. Assume $\mathcal{C}=\mathbb{Z}-\bmod A$ morphism of rings $A \rightarrow B$ is smooth if and only if

i. The ring $B$ is finitely presented in $A-$ alg.

ii. The morphism $A \rightarrow B$ is flat.

iii. The ring $B$ is a perfect complex of $B \otimes_{A} B$ modules.

The flatness of $A \rightarrow B$ is in fact equivalent to $\operatorname{Tordim}_{A} B=0$ hence the two last conditions are homological finiteness conditions. By the correspondence of Dold-Kan, the second condition can then be traduced in an homotopical condition. Finally, a result from [TV] asserts that $B$ is a perfect complex in $\operatorname{ch}\left(B \otimes_{A} B\right)$ if and only if it is homotopically finitely presentable in $s B \otimes_{A} B-\bmod$.

We provide then the category $s \mathcal{C}$ of simplicial objects in a monoidal category and all the categories $s A-\bmod , s A-a l g$ with model structures using the work of Rezk in $[\underline{\mathrm{R}}$. The classical functors between the categories $A-\bmod , A-a l g, A \in$ $s C o m m(\mathcal{C})$ induce Quillen functors between the corresponding simplicial categories. We give the following general definition for smoothness

Definition 0.2. Let $A$ be in $s \operatorname{Comm}(\mathcal{C})$, a morphism $B \rightarrow C$ in $s A-\operatorname{alg}$ is smooth if and only if

i. The simplical algebra $C$ is homotopically finitely presented in $s B-a l g$.

ii. The simplicial algebra $C$ has Tor dimension 0 on $B$.

iii. The morphism $C \otimes_{B}^{h} C \rightarrow C$ is homotopically finitely presented in $s C \otimes_{B}^{h} C-\bmod$.

The first condition imply the first condition of 0.1 ([TV], 2.2.2.4) and there is equivalence for smooth morphisms of rings. When $A, B, C$ are just rings, the Tor dimension 0 imply that the derived tensor product is weakly equivalent to the tensor product. The equivalence with previous theorem for rings is then clear.

We prove that relative smooth morphisms are stable under composition and pushouts of Algebras. We finally provide examples of smooth morphism in relative non-additive contexts, for $\mathcal{C}=\mathbb{N}-\bmod$ or $\mathcal{C}=$ Ens. In particular the affine lines $\mathbb{F}_{1} \rightarrow \mathbb{N}$ and $\mathbb{N} \rightarrow \mathbb{N}[X]$ are smooth respectively in $s \operatorname{Comm}(S e t)$ and $s \mathbb{N}-\bmod$.

\section{Preliminaries}

Let $(\mathcal{C}, \otimes, 1)$ be a complete and cocomplete closed symmetric monoidal category. In the category $\mathcal{C}$, there exists a notion of commutative monoid and for a given commutative monoid $A$, of $A$-module. Let $C o m m(\mathcal{C})$ denotes the category of commutative monoids (with unity) in $\mathcal{C}$ and for $A \in \operatorname{Comm}(\mathcal{C}), A-\bmod$ denotes the category of $A$-modules. It is well known that the category $A-\bmod$ is a closed monoidal tensored and cotensored category, complete and cocomplete. The category $\operatorname{Comm}(A-\bmod )$ will be denoted by $A-\operatorname{alg}$ and is described by the equivalence $A / C o m m(\mathcal{C}) \backsim A-a l g$. A pushout in $A-a l g$ is a tensor product in the sense that for commutative monoids $B, C \in A-a l g B \otimes A C \backsim B \coprod_{A} C$.

All along this work, $(\mathcal{C}, \otimes, 1)$ is a locally finitely presentable monoidal category i.e. verify that the (Yoneda) functor $i: \mathcal{C} \rightarrow \operatorname{Pr}\left(\mathcal{C}_{0}\right)$, where $\mathcal{C}_{0}$ is the full subcategory of finitely presented objects, is fully faithful, that $\mathcal{C}_{0}$ is stable under tensor product ans contains the unity. The functor $\operatorname{Hom}_{\mathfrak{C}}(1,-)$, denoted $(-)_{0}: X \rightarrow X_{0}$ is called "underlying set functor". For $k \in \mathcal{C}_{0}$, the functors $\operatorname{Hom}_{\mathfrak{C}}(k,-)$, denoted $(-)_{k}: X \rightarrow X_{k}$ are called "weak underlying set functor". It is known that if $\mathcal{C}$ is a locally finitely presentable monoidal category, so are its categories of modules $A-\bmod$, $A \in \operatorname{somm}(\mathcal{C})$

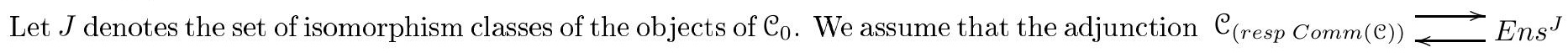
induced is monadic. Thus, on simplicial categories, the theorem of Rezk 1.2 will provide a (good) model structure. Moreover, as the forgetful functor from $A-\bmod$ to $\mathcal{C}$ preserves colimits for a monoid $A$ the functor from $A-\bmod$ to $E n s^{J}$ is also monadic. This is a consequence of the characterisation of monadic functors (see ).

There are two fundamental adjunctions:

$$
\mathrm{e} \underset{i}{\stackrel{(-\otimes A)}{\rightleftarrows}} A-\bmod \quad \mathrm{e} \underset{i}{\stackrel{L}{\rightleftarrows}} \operatorname{Comm}(\mathrm{C})
$$

where the forgetful functor $i$ is a right adjoint and the functor "free associated monoid" $L$ is defined by $L(X):=$ $\coprod_{n \in \mathbb{N}} X^{\otimes n} / S_{n}$. In these adjunctions, $\mathcal{C}$ can be replaced by $B-\bmod$ for $B \in \operatorname{Comm}(\mathcal{C})$, and $L$ by $L_{B}$ defined by $L_{B}(M):=\coprod_{n \in \mathbb{N}} M^{\otimes_{B} n} / S_{n}$. Let $\varphi\left(\operatorname{resp} \varphi_{B}\right)$ and $\psi\left(\right.$ resp $\left.\psi_{B}\right)$ denote these adjunctions for the category $\mathcal{C}($ resp $B-\bmod )$. For $X \in \mathcal{C}$ and $M \in A-\bmod , \varphi: \operatorname{Hom}_{\mathcal{C}}(X, M) \rightarrow \operatorname{Hom}_{A-\bmod }(X \otimes A, M)$ is easy to describe : 


$$
\begin{gathered}
\varphi: f \rightarrow \mu_{M} \circ I d_{A} \otimes f \\
\varphi^{-1}: g \rightarrow g \circ\left(I d_{X} \otimes i_{A}\right) \circ r_{X}^{-1}
\end{gathered}
$$

Let $s \mathcal{C}$ denotes the category of simplicial objects in $\mathcal{C}$. There is a functor "constant simplicial object" denoted $k$ from $\mathcal{C}$ to $s \mathcal{C}$ which is right adjoint to the functor $\pi_{0}$ from $s \mathcal{C}$ to $\mathcal{C}$ defined by $\pi_{0}(X):=\operatorname{Colim}(X[1] \rightleftarrows X[0])$. The tensor product of $\mathcal{C}$ induces a tensor product on $s \mathcal{C}$, its unity element is $k(1)$. For $A$ in $\operatorname{Comm}(\mathcal{C}), s A-\bmod$ and $s A-a l g$ will denote respectively the simplicial categories $s k(A)-\bmod$ and $s k(A)-a l g$. As $s C o m m(\mathcal{C}) \simeq \operatorname{Comm}(s \mathrm{C})$, we will always refer to simplicial category of commutative monoids in $s \mathcal{C}$ as $s C o m m(\mathcal{C})$. The functor induced by $L$ on simplical categories will be denoted $s L$. The functor $i: \mathcal{C} \rightarrow \operatorname{Pr}\left(\mathcal{C}_{0}\right)$ induces a functor $s i: s \mathcal{C} \rightarrow s \operatorname{Pr}\left(s \mathcal{C}_{0}\right)$.

We need finally hypotheses to endow $s \mathcal{C}, s \operatorname{Comm}(\mathcal{C})$, and for $A \in s \operatorname{Comm}(\mathcal{C}), s A-\bmod$ and $s A-a l g$ with compatible model structures. One solution of this question is to assume that the natural functors from $s \mathcal{C}$ and $s C o m m(\mathcal{C})$ to $s S e t^{J}$ are monadic, where $J$ is the set of isomorphism classes of $J$. The characterisation of monadic functors of $[\mathrm{BC}]$ implies that for any commutative simplicial monoid $A$, the induced functors from $s A-\bmod$ to $s S e t^{J}$ is also monadic.

\section{General Theory}

\subsection{Simplicial Categories and Simplicial Theories}

Definition 1.1. A simplicial theory is a monad (on $s S e t^{J}$ ) commuting with filtered colimits.

THEOREM 1.2. (Rezk)

Let $T$ be a simplicial theory in sSet ${ }^{J}$, then $T$ - alg admits a simplicial model structure. $f$ is a Weak equivalence or a fibration in $T$ - alg if and only if so is its image in sSet ${ }^{J}$ (for the projective model structure). Moreover, this Model category is right proper.

Proposition 1.3. Model structures on the simplicial categories.

i. Let $A=\left(A_{p}\right)$ be a commutative monoid in se. The monadic adjunctions $A_{p}-\bmod \longrightarrow$ Set $^{J}$ induce a monadic adjunction $s A-\bmod \longleftrightarrow s S e t^{J}$ i.e. there is an equivalence $s A-\bmod \backsim T_{A}-$ alg $=$ where $T_{A}$ is the monad induced by adjunction. In particular $s \mathcal{Q} \simeq T_{1}-$ alg.

ii. Let $A=\left(A_{p}\right)$ be a commutative monoid in se. The monadic adjunctions $A_{p}-a l g \longrightarrow$ Ens $s^{J}$ induce a monadic adjunction $s A-$ alg $\longleftrightarrow s S e t^{J}$ i.e. there is an equivalence $s A-$ alg $\simeq T_{A}^{c}-$ alg where $T_{A}^{c}$ is the monad induced by adjunction. In particular $\mathrm{sComm}(\mathcal{C}) \simeq T_{1}^{c}-$ alg.

Proof

As explained in the preliminaries, this is due to the characterisation of monadic functors $([\mathrm{Bc}])$.

Remark 1.4. The right adjoints functors all commute with filtered colimits. So do the monads which are then simplicial theories on $s S e t^{J}$.

Corollary 1.5. Let $A$ be a commutative monoid in $s \mathcal{C}$. The categories $s \mathcal{C}$ and $s A-\bmod$ and $s C o m m(\mathcal{C})$ are Model categories. Moreover, the functors $(A \otimes-)$ and $s L$ are left Quillen and their adjoints preserve by construction weak equivalences and fibrations.

Theorem 1.6. The Category se (resp sA-mod) is a monoidal model category

Proof:

The proof for $s \mathcal{C}$ and $s A-\bmod$ are similar, so let us prove it for $s \mathcal{C}$. Let $I, I^{\prime}$ be respectively the sets of generating cofibration and generating trivial cofibration. $I$ and $I^{\prime}$ are the image by the left adjoint functor respectively of generating cofibration and generating trivial cofibration in $s S e t^{J}$. We juste have to prove (cf [H] chap IV) that $I \square I$ is a set of cofibrations and $I \square I^{\prime}$ and $I^{\prime} \square I$ are sets of trivial cofibrations. It is true for generating cofibration and generating trivial cofibration in $s S e t^{J}$, which are all morphisms concentrated in a fixed level. Moreover, it is easy to verify that the functor $s K$ commutes with $\square$ of morphisms concentrated in one level. So it is true in $s \mathcal{C}$. The second axiom is clearly verified, as 1 is cofibrant. 


\subsection{Compactly Generated Model categories}

Definition 1.7. Let $\mathcal{M}$ be a cofibrantly generated simplicial model category and $I$ be the set of generating cofibrations.

i. An object $X \in I-$ cell is strictly finite if and only if there exists a finite sequence

$$
\emptyset=X_{0} \longrightarrow X_{1} \longrightarrow \cdots \longrightarrow X_{n}=X
$$

and $\forall i$ a pushout diagram:

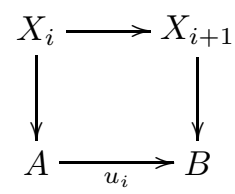

with $u_{i} \in I$.

ii. An object $X \in I-$ cell is finite if and only if it is weakly equivalent to a strictly finite object.

iii. An object $X$ is homotopically finitely presented if and only if for any filtered $\operatorname{diagram} Y_{i}$, the morphism :

$$
\operatorname{Hocolim}_{i} \operatorname{Map}\left(X, Y_{i}\right) \rightarrow \operatorname{Map}\left(X, \operatorname{Hocolim}_{i} Y_{i}\right)
$$

is an isomorphism in $\mathrm{Ho}(\mathrm{sSet})$

iv. A model category $\mathcal{M}$ is compactly generated if it is cellular, cofibrantly generated and if the domains and codomains of generating cofibration and generating trivial cofibration are cofibrant, $\omega$-compact and $\omega$-small (Relative to $\mathcal{M}$ ).

Proposition 1.8. Let $\mathcal{M}$ be a compactly generated model category.

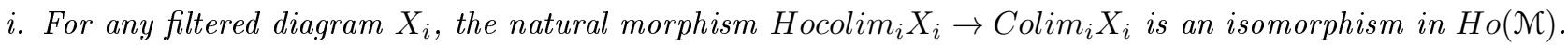

ii. Assume that filtered colimits are exact in $\mathcal{M}$. Then homotopically finitely presented objects in $\mathcal{M}$ are exactly objects equivalent to weak retracts of strictly finite $I$ - cell objects.

Proposition 1.9. $\quad$ i. The simplicial model category sSet ${ }^{J}$ is compactly generated.

ii. The categories of simplicial algebras over a simplicial theory are compactly generated.

Lemma 1.10. Let $A$ be in $s \operatorname{Comm}(\mathcal{C})$. Let $u^{j}$ be the family of images by the left adjoint functor in $s A-m o d$ (resp $s A-$ alg) of elements $*_{j}$ of $s S e t^{J}$ defined by $*$ on level $j$ and $\emptyset$ on other levels. Any codomains of a generating cofibrations of $s A-\bmod \left(r e s p s A-\right.$ alg) is weakly equivalent to an object $u^{j}$. Any domain of a generating cofibration is weakly equivalent, for a given element $j$ in $J$, to an object obtained from the initial object (denoted $\emptyset)$ and $u^{j}$ in a finite number of homotopic pushouts.

Proof:

Generating cofibrations of $s A-\bmod$ are images of generating cofibrations of $s S e t^{J}$ by the left adjoint functor. Generating cofibrations of $s$ Set are morphisms $\delta \Delta^{p} \rightarrow \Delta^{p}$. Their codomain is contractible, thus so are the codomains of generating cofibrations of $s$ Set $^{J}$ for the projective model structure, and their image by by the left adjoint is weakly equivalent to the unity 1 . For the domains, consider the relation $\delta \Delta^{p+1} \simeq \Delta^{p+1} \coprod_{\delta \Delta^{p}}^{h} \Delta^{p+1} \backsim * \coprod_{\delta \Delta^{p}}^{h} *$ and $\delta \Delta^{0}=\emptyset$. Domains of generating cofibration in $s S e t^{J}$ for the projective model structure are objects $\left(\delta \Delta^{p, j}\right)_{p \in N, j \in V}$ defined in level $i \neq j$ by $\emptyset$ and in level $j$ by $\delta \Delta^{p}$ and verify the relation

$$
\left(\delta \Delta^{p, j}\right) \simeq *_{j} \coprod_{\left(\delta \Delta^{p-1, j}\right)}^{h} *_{j}
$$

Clearly $\delta \Delta^{0, j}=\emptyset$ and $\delta \Delta^{1, j}=*_{j}$. Let $u^{p, j}$ denote the image of $\delta \Delta^{p, j}$. For all $j, u^{p, j}$ is obtained in a finite number of pushouts from $\emptyset$ and $u^{j}$.

Corollary 1.11. of proposition 1.9 and lemma 1.10 ,

i. The Simplicial Model categories $s \mathcal{C}, s A-\bmod (A \in s \operatorname{Comm}(\mathcal{C})), s C o m m(\mathcal{C})$ and $s A-\operatorname{alg}(a \in s C o m m(\mathcal{C}))$ are compactly generated.

ii. Homotopically finitely presented objects of $s$ - mod (resps A-alg) are exactly objects weakly equivalent to weak retracts of strictly finite $I-$ Cell objects. 
iii. The sub-category of $\mathrm{Ho}(s s A-\bmod )(\operatorname{resp~} \mathrm{Ho}(s A-a l g)) H o(s A-\bmod )_{c}\left(\mathrm{resp} H o(s A-a l g)_{c}\right)$ Consisting of homotopically finitely presented objects is the smallest full sub-category of $\mathrm{Ho}(\mathrm{sA}-\mathrm{mod})$ (resp $\mathrm{Ho}(\mathrm{sA}-\mathrm{alg})$ ) containing the family $\left(u^{1, j}\right)_{j \in V}\left(\right.$ resp $\left.\left(u_{a}^{1, j}\right)_{j \in V}\right)$, and stable under retracts and homotopic pushouts.

Proof of $i i i$ :

Let $\mathcal{D}$ be the smallest full sub-category of $H o(s \mathcal{C})$ containing $\left(u^{j}\right)_{j \in V}\left(r e s p\left(u_{A}^{j}\right)_{j \in V}\right)$, the initial object $\emptyset$ and stable under retracts and homotopic pushouts. Clearly, by $i i$, as $\left(\emptyset \rightarrow u^{j}\right)_{j \in V}$ are generating cofibrations of $s \mathcal{C}, H o(s \mathcal{C})_{c}$ contains the family $\left(u^{j}\right)_{j \in V}$, and is stable under retracts and homotopic pushouts. Thus $\mathcal{D} \subset H o(s \mathcal{C})_{c}$. Reciprocally, let $X$ be an object of $H o(s \mathrm{C})_{c}$, by $i i, X$ is isomorphic to a weak retract of a strictly finite $I-$ cell object. Therefore, there exists $n$ and $X_{0} \ldots X_{n}$ such that:

$$
\emptyset=X_{0} \longrightarrow X_{1} \longrightarrow \cdots \longrightarrow X_{n}=X
$$

and $\forall j \in\{0, . ., n-1\}, \exists K \rightarrow L$, a generating cofibration such that:

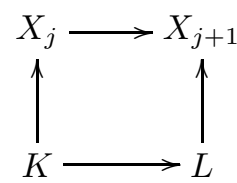

is a pushout diagram. Now, as domains and codomains of generating cofibrations are in $\mathcal{D}, X$ is in $\mathcal{D}$.

\subsection{Categories of Modules and Algebras}

Proposition 1.12. Let $A$ be in $s C o m m(\mathcal{C})$ and $B$ be a simplicial monoid in $s A-$ alg, cofibrant in $s A-\bmod$.

$i$. The forgetful functor from $s B-\bmod$ to $s A-\bmod$ preserves cofibrations

ii. The forgetful functor from $s A-$ alg to $s A$ - mod preserves cofibrations whose domain is cofibrant in sA-mod. In particular, it preserves cofibrant objects.

Proof

In each case, we just have to prove it for generating cofibrations and then generalize it to any cofibration by the small object argument.

Proof of $i$ : First, we choose a generating cofibration in $s B-\bmod$. As generating cofibration of $s B-\bmod$ are images of generating cofibrations of $s S e t^{J}$, we set $L \rightarrow M$, a generating cofibration in $s S e t^{J}$. Let $K_{A}, K_{A}$ denotes respectively the left adjoint functors (from $s S e t^{J}$ ) for $s A-\bmod$ and $s B-\bmod$. The axiom of stability under $\square$ implies that the morphism $(\emptyset \rightarrow B) \square\left(K_{A}(L) \rightarrow K_{A}(M)\right)$ is a cofibration in $s A-\bmod$. This morphism is in fact $K_{B}(L)=B \otimes_{A} K_{A}(L) \rightarrow B \otimes_{A} K_{A}(M)=K_{B}(M)$, hence generating cofibrations of $s B-$ mod are cofibrations in $s A-\bmod$.

Proof of $i i$ : As for $i$, let $N \rightarrow M$ be a generating cofibration in $s S e t^{J}$. Let $L^{s}$ denotes the functor "free associated commutative monoid" of $s S e t^{J}$. The functors $L$ (resp $s L_{A}$ in $s A-\bmod$ ) and $K_{A}$ are defined by colimits and so commute up to isomorphisms. That means that $K_{A} \circ L^{s} \simeq s L_{A} \circ K_{A}$. So the generating cofibration of $s A-$ alg corresponding to $N \rightarrow M$ is isomorphic to $K_{A}(L(N)) \rightarrow K_{A}(L(M))$. To prove that it is a cofibration in $s A-\bmod$, we have then to prove that the morphism $L(N) \rightarrow L(M)$ is injective levelwise and this is clear as for any morphism $N^{\otimes n} / S_{n} \rightarrow M^{\otimes n} / S_{n}$ is injective. Thus any generating cofibration of $s A-a l g$ is a cofibration in $s A-\bmod$. In fact it is a generating cofibration of $s A-\bmod$. To use the small object argument (of $s A-a l g$ ), we need to verify that it preserves cofibrations in $s A-\bmod$. In fact, we need to check that an homotopic pushout in $s A-a l g$ of a cofibration in $s A-\bmod$ is still a cofibration in $s A-\bmod$. We let the reader verify that it is a consequence of the axiom of stability by $\square$. Finally, the forgetful functor preserves cofibrations and, as $A$ is cofibrant in $s A-\bmod$, any cofibrant object of $s A-$ alg is also cofibrant in $s A-\bmod$.

Lemma 1.13. Let $A \rightarrow B \in s C o m m(\mathcal{C})$ be a trivial cofibration between cofibrant objects. The categories of module are equivalent i.e. $H o(s A-\bmod ) \simeq H o(s B-\bmod )$.

Proof:

We must prove that for $X$ cofibrant in $s A-\bmod$ and $y$ fibrant in $s B-\bmod , \varphi_{a}(f): x \otimes_{A} B \rightarrow y$ is a weak equivalence in $s B-\bmod$ if and only if so is $f: X \rightarrow Y$ in $s A-\bmod$. By previous lemma, $A \rightarrow B$ is a trivial cofibration in $s A-\bmod$. Thus as $X$ is cofibrant, using the axiom of stability under $\square, g: X \rightarrow B \otimes_{A} X$ is a weak equivalence in $s A-\bmod$. By construction of the adjunction $\varphi_{A}$, the following diagram is commutative : 


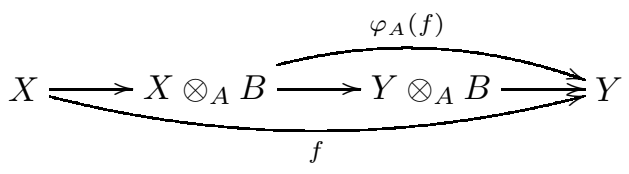

Thus $f=g \circ \varphi_{A}(f)$. Finally, $\varphi_{A}(f)$ is a weak equivalence in $s A-\bmod$ if and only if so it is in $s B-\bmod$ and the $t w o$ out of three axiom ends the proof.

Proposition 1.14. Let $f: A \rightarrow B \in s \operatorname{Comm}(\mathcal{C})$ be a weak equivalence between cofibrant objects. The categories of module are equivalent ie $H o(s A-\bmod ) \simeq H o(s B-\bmod )$.

Let $r_{c}$ be the fibrant replacement of $s \operatorname{Comm}(\mathcal{C})$, then by previous lemma, the homotopical categories of modules over $A$ and $r_{c} A$ (resp $B$ and $r_{c} B$ ) are equivalent. Thus $A$ and $B$ can be taken fibrant and $f$ is an homotopy equivalence i.e. $\exists g$ such that $f \circ g$ and $g \circ f$ are homotopic to identity. The following diagrams are commutative:
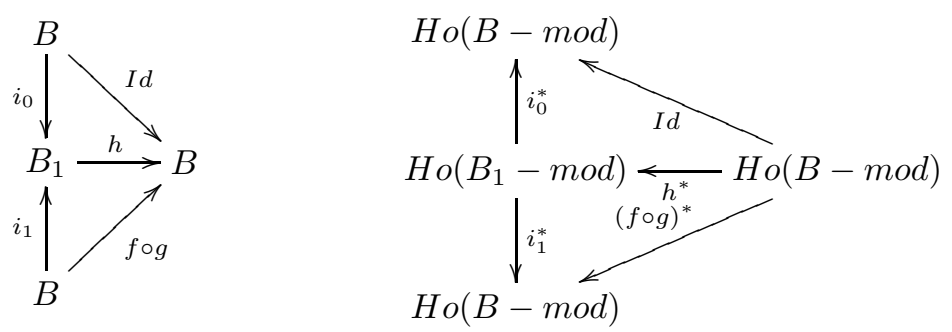

where $i_{0}$ and $i_{1}$ are cofibrations and have the same right inverse $p$ i.e. such that $p \circ i_{1}=p \circ i_{0}=I d_{B}$. the morphism $h$ is a trivial fibration thus $i_{0}$ is a weak equivalence. By previous lemma, $i_{0}^{*}$ is an equivalence of categories. Thus so is $p^{*}$. As $i_{1}^{*}$ and $i_{0}^{*}$ are both inverses of $p^{*}$, they are isomorphic and $i_{1}^{*}$ is also an equivalence. Finally, $h^{*}$ is an equivalence and so is $(f \circ g)^{*}$. The same method prove that $(g \circ f)^{*}$ is an equivalence.

\subsection{Finiteness Conditions}

Definition 1.15. Let $q_{c}$ be a cofibrant replacement in $s \operatorname{Comm}(\mathcal{C})$ and $f: A \rightarrow B$ be a morphism in $s C o m m(\mathcal{C})$.

$\triangleright$ The morphism $f$ is homotopically finite (denoted $h f$ ) if $B$ is homotopically finitely presented in $s q_{c} A-\bmod$.

$\triangleright$ The morphism $f$ is homotopically finitely presented (denoted $h f p$ ) if $B$ is homotopically finitely presented in $s q_{c} A-a l g$.

Remark 1.16. The morphism $A \rightarrow B$ is $h f$ (resp $h f p$ ) if and only if the morphism $q_{c} A \rightarrow q_{c} B$ is $h f$ (resp $h f p$ ). The morphism $q_{c} B \rightarrow B$ is always $h f$.

Lemma 1.17. The $h f$ (resp hfp) morphisms are stable under composition.

Proof

The proofs for $h f$ morphisms and $h f p$ morphisms are analogous so let us prove it for $h f$ morphisms. Let $A \rightarrow B \rightarrow C$ be the composition of two hf morphisms. There is a diagram

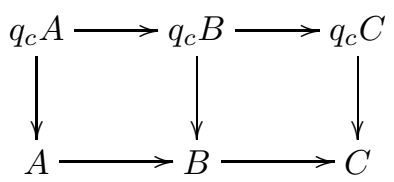

and forgetful functors $F_{1}: s q_{c} C-\bmod \rightarrow s q_{c} B-\bmod$ and $F_{2}: s q_{c} B-\bmod \rightarrow s q_{c} A-\bmod$. The image $F_{1}\left(q_{c} C\right)$ of $q_{c} C$ is homotopically finitely presented in $s q_{c} B-\bmod$ hence weakly equivalent to a retract of a finite homotopical colimit of $q_{c} B$ in $H o\left(s q_{c} B-\right.$ mod $)$. The forgetful functor $F_{2}$ preserves retracts, equivalences, finite colimits, cofibrant objects and cofibrations whose domain is cofibrant. Thus it also preserves finite homotopical colimit and sends $q_{c} C$ to a retract of a finite homotocipal colimit of $q_{c} B$ in $H o\left(s q_{c} A-\bmod \right)$. As $q_{c} B$ is homotopically finitely presented in $s q_{c} A-\bmod$, and as homotopically finitely presented objects are stable under retracts, equivalences and finite homotopical colimit, $C$ is sent by $F_{2} \circ F_{1}$ in $s q_{c} A-\bmod _{c}$. Hence $A \rightarrow C$ is finite. 
Lemma 1.18. The hf (resp hfp) morphims are stable under homotopic pushout of simplicial monoids.

Proof:

The proofs for $h f$ morphisms and $h f p$ morphisms are analogous so let us prove it for $h f$ morphisms. Let $A \rightarrow B$ and $A \rightarrow C$ be in $s C o m m(\mathcal{C})$ such that the first is finite. Let $q_{c A}$ be the cofibrant replacement of $q_{c} A-a l g$, it is weakly equivalent to $q_{c}$ and the object $q_{c_{A}} B$ is homotopically finitely presented in $s q_{c} A-\bmod$. Let us prove that $B \otimes_{A}^{h} C \simeq q_{c A} B \otimes_{q_{c} A} q_{c} C$ (in $H o\left(q_{c} A-\right.$ mod), Reedy lemma) is homotopically finitely presented in $q_{c} C-$ mod. The forgetful functor $s q_{c} C-\bmod \rightarrow s q_{c} A-\bmod$ preserves filtered colimits and weak equivalences hence it preserves homotopical filtered colimits. Thus the derived functor $-\otimes_{q_{c} A} q_{c} C$ preserves homotopically finitely presented objects. So $B \otimes_{A}^{h} C$ is homotopically finitely presented in $H o\left(q_{c} C\right)$.

\subsection{A Definition for Smoothness}

Definition 1.19. A morphism $A \rightarrow B$ in $s \operatorname{Comm}(\mathcal{C})$ is formally smooth if the morphism $B \otimes_{A}^{h} B \rightarrow B$ is $h f$.

Remark 1.20. This definition does not generalise the definition of formal smoothness in the sense of rings. However, the corresponding notion of smoothness is a generalisation of the classical notion of smoothness, as it will be proved in this article.

Proposition 1.21. Formally smooth morphisms are stable under composition.

Proof:

by previous remarks, it can be assumed that $A$ is cofibrant in $s C o m m(\mathcal{C}), B$ is cofibrant in $s A-a l g$ and $C$ is cofibrant in $s B-$ alg. Let $A \rightarrow B \rightarrow C$ be the composition of two formally smooth morphisms. The morphisms $B \amalg_{A} B \rightarrow B$ and $C \coprod_{B} C \rightarrow C$ are $h f$. The following diagram commutes and is clearly cocartesian :

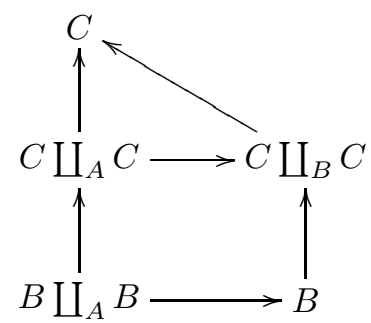

Thus, if it is cofibrant for the Reedy stucture, it will be homotopically cocartesian. The morphisms $B \backsim B \coprod_{A} A \rightarrow$ $B \coprod_{A} B$ and $B \coprod_{A} B \rightarrow C \coprod_{A} C$ are images by the left Quillen functor colim, of clear Reedy cofibrations (see [A] for a descriptions of these cofibrations), thus are cofibrations. In particular $B \coprod_{A} B$ and $C \coprod_{A} C$ are cofibrant and the diagram considered is Reedy cofibrant. Finally, the morphism $C \coprod_{A} C \rightarrow C \coprod_{B} C$ is $h f$ as a pushout of $h f$ morphisms and $C \amalg_{A} C \rightarrow C$ is $h f$ as a composition of $h f$ morphisms.

Proposition 1.22. Formally smooth morphism are stable under homotopic pushout.

Proof:

Let $u: A \rightarrow B$ be a formally smooth morphism and $C$ be a commutative $A$-algebra. By previous remarks it can be assumed that $A$ and $c$ are cofibrants in $s \operatorname{Comm}(\mathcal{C})$ and that $B$ is cofibrant in $s A-a l g$. Let $D$ denote the homotopic pushout of $B \otimes_{A} C$ and $u^{\prime}$ denote the morphism from $B$ to $D$. Clearly:

$$
D \otimes_{C} D \simeq B \otimes_{A} C \otimes_{C} B \otimes_{A} C \simeq B \otimes_{A} D
$$

Thus the following diagram commutes :

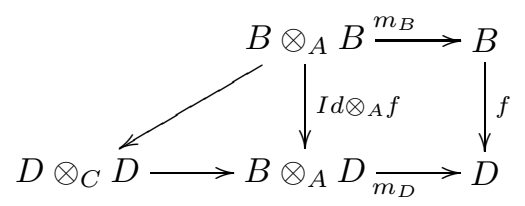

And is cocartesian :

$$
B \otimes_{B \otimes_{A} B} B \otimes_{A} B \otimes_{A} C \backsim B \otimes_{A} C \backsim D
$$


Moreover it is clearly cofibrant as $B \otimes_{A}-$ preserve cofibrations. Finally by stability of $h f$ morphism under homotopic pushouts, the morphism $C \rightarrow D$ is formally smooth.

Definition 1.23. Let $A$ be in $s \operatorname{Comm}(\mathcal{C})$ and $M$ be in $s A-\bmod$.

i. The object $M$ is $n$-truncated if $\operatorname{Map}_{s} \mathrm{e}(X, M)$ is $n$-truncated in $s S e t, \forall X \in s \mathcal{C}$.

ii. The Tor-Dimension of $M$ in $s A-\bmod$ is defined by

$$
\operatorname{Tordim}_{A}(M)=\inf \left\{n \text { st } M \otimes_{A}^{h} X \text { is } n+p-\text { truncated } \forall X \in s A-\bmod p-\text { truncated }\right\}
$$

iii. A morphism of monoids $A \rightarrow B$ has Tor dimension $n$ if $\operatorname{Tordim}_{A}(B)=n$.

Lemma 1.24. Tor dimension zero morphisms are stable under composition and homotopic pushout.

Proof:

Let $A \rightarrow B \rightarrow C$ be the composition of two Tor dimension zero morphisms. Let $M$ be a $p$ truncated $A$-module,

$$
M \otimes_{A} C \simeq M \otimes_{A} B \otimes_{B} C .
$$

As $\operatorname{Tordim}_{A}(B)=0, M \otimes_{A} B$ is a $p$ truncated $B$-module. As $\operatorname{Tordim}_{B}(C)=0, M \otimes_{A} C$ is a $p$ truncated $C$-module. Thus Tordim $_{A}(C)=0$.

Let $A \rightarrow B$ be a Tor dimension zero morphism and $A \rightarrow C$ be a morphism in $C o m m(\mathcal{C})$. Let $M$ be in $C$-mod and let $D$ denote the pushout $B \otimes_{A} C$. We have

$$
M \otimes_{C} B \otimes_{A} C \backsim M \otimes_{A} B .
$$

Thus, $\operatorname{Tordim}_{A}(B)=0$ implies $\operatorname{Tordim}_{C}(D)=0$.

Definition 1.25. A morphism $A \rightarrow B$ in $\operatorname{Comm}(\mathcal{C})$ is smooth if it is formally smooth, $h f p$ and has Tor-Dimension zero. A morphism of affine scheme is smooth if the corresponding morphism of monoids is smooth. We say that an affine scheme $X$ is smooth if the morphism $X \rightarrow \operatorname{Spec}(1)$ is smooth.

THEOREM 1.26. Smooth morphisms are stable under composition and homotopic pushout.

Proof:

This a corollary of $1.24,1.21,1.22,1.17$ and 1.18

\section{Simplicial Presheaves Cohomology}

In the article [T1], B. Toën define a cohomology for a connected and pointed simplicial presheaf. We will define here a cohomology for a general simplicial presheaf. This theory will be used to fin examples of smooth morphisms of commutative monoids (in sets). The references cited in this section are [T1], [GJ] and [J].

\subsection{Definitions}

In this section, $\mathcal{D}$ is a category and $\operatorname{sPr}(\mathcal{D})$ is the category of simplicial presheaves over $\mathcal{D}$.

Definition 2.1. ([GJ] $V I .3)$

Soit $F \in s \operatorname{Pr}(\mathcal{D})$. The tower of $n$-truncations of $F$ is a Postnikov tower:

$$
\cdots \longrightarrow \tau_{\leq n} F \longrightarrow \tau_{\leq n-1} F \longrightarrow \tau_{\leq 1} F \longrightarrow \tau_{\leq 0} F
$$

Definition 2.2. Let $F$ be a simplicial presheaf.

$\triangleright$ The functor $\pi_{0}(F): \mathcal{D} \rightarrow$ Ens is defined by $\pi_{0}(F): X \rightarrow \pi_{0}(F(X))$.

$\triangleright$ The category $(\mathcal{D} / F)_{0}$ is the full subcategory of $s \operatorname{Pr}(\mathcal{D}) / F$ whose objects are in $\mathcal{D}$.

$\triangleright$ The functor $\pi_{n}(F):(\mathcal{D} / F)_{0} \rightarrow$ Ens is defined by $\pi_{n}(F)(X, u)=\pi_{n}(F(X), u)$.

Definition 2.3. Let $G$ be a simplicial group. 
$\triangleright$ The bisimplicial set $E(G, 1)$ is defined by $E(G, 1)_{p, q}=G_{p}^{q}$.

$\triangleright$ The classifying space of $G$, denoted $K(G, 1)$, is given by the diagonal of the bisimplicial set $E(G, 1) / G$. More precisely $K(G, 1)_{n}=G_{n}^{n} / G_{n}$. It is abelian if $G$ is abelian.

$\triangleright$ The endofunctor of abelien groups $K(G, 1)^{\circ n}$ is denoted $K(G, n)$.

Remarks 2.4. As the diagonal of $E(G, 1)$ is pointed (by identity), the simplicial set $K(G, 1)$ is also pointed. In particular, $\pi_{n}(K(G, 1), *) \simeq \pi_{n-1}\left(G, e_{g}\right)$. This construction is functorial (in $G$ ) and then extends to presheaves of simplicial groups.

\subsection{Simplicial presheaves Cohomology}

It is necessary to work in the proper category to construct a cohomology for a simplicial presheaf $F$ which is not connected or pointed. In fact the 1-truncation of $F$ is the nerve $N G$ of a groupoid $G$ and in the category $s P r(\mathcal{D}) / N G$, $F$ becomes connected and pointed. But in this category, there is no clear construction fro classifying spaces. The solution of this problem is given by a Quillen equivalence with the category $s \operatorname{Pr}(\mathcal{D} / G)$, for a well chosen category $\mathcal{D} / G$. We choose now a simplicial presheaf $F$.

\section{The Category of Presheaves}

\section{The left adjoint functor $\widetilde{(-)}$}

Definition 2.5. The category $\mathcal{D} / G$ is the category whose objects are couples $(X, x), x: X \rightarrow N G$, and whose morphisms from $(X, x)$ to $(Y, y)$ are couples $(f, u)$ where $f: X \rightarrow Y$ and $u: y \circ f \simeq x$ in $G(X) \simeq \pi_{1} F(X)$.

Next step is to construct a functor $\widetilde{(-)}: \mathcal{D} / G \rightarrow \operatorname{ser}(\mathcal{D} / N G)$.

Definition 2.6. Let $(X, x$,$) be in \mathcal{D} / G$. Define a presheaf of groupoïds $G_{X, x}$ on $\mathcal{D}$. Ths image of $S \in \mathcal{D}$ is the groupoid described as follow

- The objects are triples $(u, y, h), u: S \rightarrow X, y \in G(S)$, and $h: x \circ u \bumpeq y \in G(S)$.

- A morphism from $(u, y, h)$ to $\left(u^{\prime}, y^{\prime}, h^{\prime}\right)$ is an endomorphism $k$ of $S$ such that $k^{*}(h: x \circ u \rightarrow y)=h^{\prime}: x^{\prime} \circ u^{\prime} \rightarrow y^{\prime}$.

Let $\breve{X}$ denote the nerve of this groupoid.

Remark 2.7. There is a commutative diagram of presheaves of groupoids

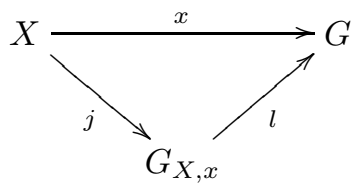

where $l$ is the projection on $G$ and $j$ is given for $S \in \mathcal{D}$ by $j(S): u \in \operatorname{Hom}_{\mathcal{D}}(S, X) \rightarrow(u, x \circ u, I d) \in G_{X, x}$.

Applying the functor nerve, one get a morphism $\breve{x}:=N l: \breve{X} \rightarrow G$. It defines a functor

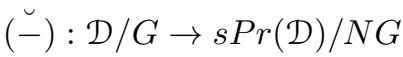

$$
\begin{aligned}
& (X, x) \rightarrow(\breve{X}, \breve{x})
\end{aligned}
$$

Definition 2.8. The functor $\widetilde{(-)}: \mathcal{D} / G \rightarrow s \operatorname{Pr}(\mathcal{D}) / N G$ is defined by

$$
\widetilde{(-)}:(X, x) \rightarrow(\widetilde{X}, \widetilde{x}):=Q(\breve{X}, \breve{x})
$$

where $Q$ is a cofibrant replacement in $\operatorname{ser}(\mathcal{D}) / N G$.

Remarks 2.9. This functor has a kan extension to $\operatorname{ser}(\mathcal{D} / G)$, still denoted

$$
\widetilde{(-)}: \operatorname{sPr}(\mathcal{D} / G) \rightarrow \operatorname{Spr}(\mathcal{D}) / N G \text {. }
$$

In facts, the category $s \operatorname{Pr}(\mathcal{D} / G)$ is equivalent to the category $s \operatorname{Pr}(\mathcal{D})^{N G}$ defined in $[\mathrm{J}]$ and the equivalence of category we are constructing is constructed in a different way and a more general situation in $[\mathrm{J}]$. 
The right adjoint functor $(-)_{1}$ We construct now the (right) adjoint of $\widetilde{(-)}$, denoted $(-)_{1}$.

Definition 2.10. The functor $(-)_{1}: s \operatorname{Pr}(\mathcal{D}) / N G \rightarrow s \operatorname{Pr}(\mathcal{D} / G)$ is defined by

$$
(-)_{1}:(H, u) \rightarrow H_{1}:=(X, x) \rightarrow \underline{H o m}_{s P r}^{\Delta}(\mathcal{D}) / N G((\widetilde{X}, \widetilde{x}),(H, u))
$$

where $\underline{H o m}^{\Delta}$ is the simplicial Hom. As $(\widetilde{X}, \widetilde{x})$ is constructed cofibrant, the functor $(-)_{1}$ is right Quillen and its adjoint is then left Quillen. We prove now that $R(-)_{1}$ commute with homotopy colimits. We need to recall first some properties.

Definition 2.11. Let $(H, h)$ be in $\operatorname{ser}(\mathcal{D}) / N G$ and $(X, x)$ be in $\mathcal{D} / G$. Define an object $\left(H_{X}, h_{x}\right)$ by the homotopy pullback diagram

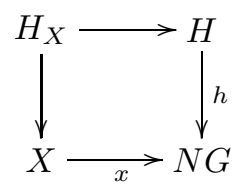

Lemma 2.12. Let $(H, f)$ be an homotopy colimit, $H \simeq \operatorname{Hocolim}\left(H_{i}\right)$, in $\operatorname{sPr}(\mathcal{D}) / N G$ and let $(X, x)$ be in $\mathcal{D} / G$.

$\diamond$ There is an isomorphism $H_{X} \simeq \operatorname{Hocolim}\left(H_{i}\right)_{X}$ in $\operatorname{Ho}(\operatorname{sPr}(\mathcal{D}) / N G)$.

$\diamond$ There is an isomorphism $R H_{1}(X) \simeq \operatorname{Map} \operatorname{spr}(\mathcal{D}) / X\left((X, I d),\left(H_{X}, h_{x}\right)\right)$.

Corollary 2.13. The functor $R(-)_{1}$ commute with homotopy colimits.

Proof:

Let $H$ be isomorphic to $\operatorname{Hocolim}\left(H_{i}\right)$ and $(X, x)$ be in $\mathcal{D} / G$.

$$
\begin{gathered}
R H_{1}(X) \simeq \operatorname{Map}_{\operatorname{PPr}(\mathcal{D}) / X}\left((X, I d),\left(\left[\operatorname{Hocolim}\left(H_{i}\right)\right]_{X},\left[\operatorname{Hocolim}\left(h_{i}\right)\right]_{x}\right)\right) \\
\simeq \operatorname{Map}_{\operatorname{sPr}(\mathcal{D}) / X}\left((X, I d),\left(\operatorname{Hocolim}\left[\left(H_{i}\right)_{X}\right], \operatorname{Hocolim}\left[\left(h_{i}\right)_{x}\right]\right)\right) \simeq \operatorname{Hocolim}\left(R\left(H_{i}\right)_{1}(X)\right)
\end{gathered}
$$

\section{The Equivalence}

Proposition 2.14. The Quillen functors $\widetilde{(-)}$ and $(-)_{1}$ define a Quillen equivalence.

Proof:

The functor $\widetilde{(-)}$ commutes with homotopy colimits and as any object in $\operatorname{ser}(\mathcal{D} / G)$ is an homotopy colimit of representable objects $H \simeq \operatorname{Hocolim}\left(X_{i}\right)$, its image can be computed in terms of representable objects, i.e. $\widetilde{H} \backsim$ $\operatorname{Hocolim}\left(\widetilde{X_{i}}\right)$. The short exact sequence $H_{1} \rightarrow H \rightarrow \tau_{\leq 1} H$ proves that $(-)_{1}$ preserves weak equivalences. Then

$$
(\widetilde{H})_{1} \simeq\left(\operatorname{Hocolim}\left(\widetilde{X}_{i}\right)\right)_{1} \simeq \operatorname{Hocolim}\left(X_{i}\right) \simeq H .
$$

If $H$ is cofibrant in $s \operatorname{Pr}(\mathcal{D} / G)$ and $H^{\prime}$ is fibrant in $s \operatorname{Pr}(\mathcal{D}) / N G$, we consider a morphism between short exact sequences

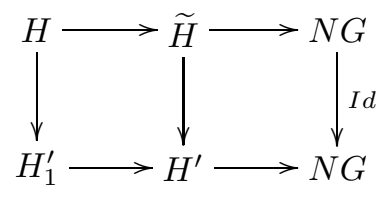

Applying the functors $\pi_{i}$, it is clear that if $\widetilde{H} \rightarrow H^{\prime}$ is an equivalence, so is $H \rightarrow H_{1}^{\prime}$ and reciprocally, if $H \rightarrow H_{1}^{\prime}$, the homotopic fibers of $\widetilde{H} \rightarrow H^{\prime}$ upon $N G$ are equivalences thus so is $\widetilde{H} \rightarrow H^{\prime}$. 


\section{The Cohomology}

Definition 2.15. Let $F$ be in $\operatorname{sPr}(\mathcal{D})$, a local system on $F$ is a presheaf of abelian groups on $\mathcal{D} / G$, where $G$ verifies $N G \backsim \tau_{\leq 1} F$. A Morphism of local system is a morphism of presheaves of abelian groups. The category of local systems on $F$ will be denoted $\operatorname{sysloc}(F)$. The n-th classifying space of $M$ is denoted $K(M, n)$ and its image by $\widetilde{L(-)}$ is denoted $L \tilde{K}(M, n)$.

Remark 2.16. The object $L \tilde{K}(M, n)$ is characterised up to equivalence by the fact that $\pi_{n}(L \tilde{K}(M, n)) \simeq M, \pi_{1}(L \tilde{K}(M, n)) \simeq$ $\pi_{1}(F), \pi_{0}(L \tilde{K}(M, n)) \simeq \pi_{0}(F)$ and that its other homotopy presheaves of groups are trivial.

Definition 2.17. Let $F$ be in $s \operatorname{Pr}(\mathcal{D})$ and $M$ be a local system on $F$. The n-th cohomology group of $F$ with coefficient in $M$ is

$$
H^{n}(F, M):=\pi_{0} \operatorname{Map}_{\operatorname{PPr}(\mathcal{D}) / N G}(F, L \tilde{K}(M, n))
$$

The standard example of local system is $\pi_{n}$. Indeed, it has been defined on $(\mathcal{D} / F)_{0}$ but it clearly lifts to $\mathcal{D} / G$.

The important theorem is here.

TheOREM 2.18. Let $G$ be a grouoid. For all $m$, the functor

$$
\begin{gathered}
H^{m}(N G,-): \operatorname{Sysloc}(N G) \rightarrow A b \\
M \rightarrow H^{m}(N G, M)
\end{gathered}
$$

is isomorphic to the $n$-th derived functor of the functor $H^{0}(N G,-)$.

Proof:

There is an equivalence between the category of simplicial abelian group presheaves, denoted $s A b(\mathcal{D} / G)$, on $\mathcal{D} / G$ and the category of complexes of abelian group presheaves with negative or zero degree, denoted $C^{-}(\mathcal{D} / G, A b)$. This is a generalisation of Dold-Kan correspondence. There is a correspondence between quasi-isomorphism of complexes and weak equivalences of simplicial presheaves, and then an induced equivalence between the homotopical categories :

$$
\Gamma: D^{-}(\mathcal{D} / G, A b) \simeq H o(s A b(\mathcal{D}) / G)
$$

The derived functors of $H^{0}$ are then given by

$$
H_{\text {der }}^{m}(\mathcal{D} / G, M) \simeq \operatorname{Hom}_{D^{-}(\mathcal{D} / G, A b)}(\mathbb{Z}, M[m])
$$

Where $\mathbb{Z}$ is regarded as a complex concentrated in degree zero and $M[m]$ is concentrated in degree $-m$, with value $M$. As $\Gamma(\mathbb{Z})$ is the constant presheaf with fiber $\mathbb{Z}$, still denoted $\mathbb{Z}$, and as $\Gamma(M[m])$ is equivalent to $K(M, m)$, $\Gamma$ induces an isomorphism :

$$
\operatorname{Hom}_{D^{-}(\mathcal{D} / G, A b)}(\mathbb{Z}, M[m]) \bumpeq \operatorname{Hom}_{H o(s A b(\mathcal{D} / G))}(\mathbb{Z}, K(M, m))
$$

Finally, the adjunction between the abelianisation functor, denoted $\mathbb{Z}(-)$ from $s \operatorname{Pr}(\mathcal{D} / G)$ to $s A b(\mathcal{D} / G)$ and the forgetful functor gives

$$
\operatorname{Hom}_{D^{-}(\mathcal{D} / G, A b)}(\mathbb{Z}, M[m]) \simeq \operatorname{Hom}_{H o(s P r(\mathcal{D} / G))}(*, K(M, m)) \simeq H^{m}(N G, M) .
$$

\section{Obstruction Theory}

There is an homotopic pullback diagram in $\operatorname{ser}(\mathcal{D} / G)$ :

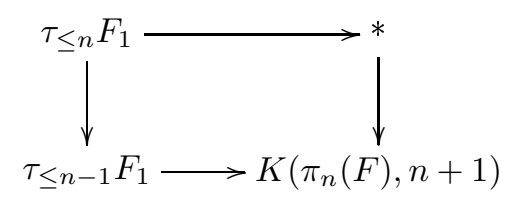

As $F_{1}$ is 1-connex, this pullback diagram is a (functorial) generalisation to presheaf of the diagram given by the proposition 5.1 of [GJ]. By the quillen equivalence $\left((-)_{1}, \widetilde{(-)}\right)$, there is an homotopic pullback diagram: 


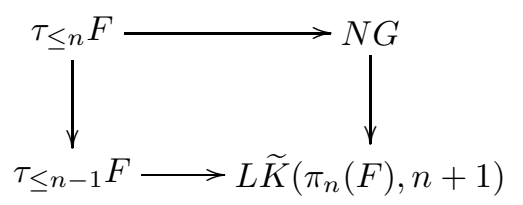

If $H \rightarrow \tau_{\leq n-1} F$ is a morphism in $\operatorname{Ho}(s \operatorname{Pr}(\mathcal{D}) / N G)$, it has a lift to $\tau_{\leq n} F$ if and only if it is send to a zero element in the group

$$
\pi_{0} \operatorname{Map} \operatorname{Pr}(\mathcal{D}) / N G\left(H, L \widetilde{K}\left(\pi_{n}(F), n+1\right)\right)
$$

This group can be described in terms of cohomology. Indeed, if $G^{\prime}$ is a groupoid such that $N G^{\prime} \backsim \tau<1$. Let $u$ denote the morphism $u: N G^{\prime} \rightarrow N G$. To simplify the notations, we still write $H$ for what we sould call $u^{*} H$. There is a Quillen adjunction:

$$
\operatorname{sPr}(\mathcal{D}) / N G_{-\mathrm{C}_{N G}^{\prime} N G^{\prime}}^{\stackrel{u^{*}}{\longleftrightarrow}} \mathcal{D} / N G
$$

which induces an isomorphism

$$
M a p_{s P r(\mathcal{D}) / N G}\left(H, L \widetilde{K}\left(\pi_{n}(F), n+1\right)\right) \simeq \operatorname{Map}_{s \operatorname{Pr}(\mathcal{D}) / N G^{\prime}}\left(H, L \widetilde{K}\left(\pi_{n}(F), n+1\right) \times_{N G} N G^{\prime}\right) .
$$

There is a clear weak equivalence $L \widetilde{K}\left(\pi_{n}(F), n+1\right) \times_{N G} N G^{\prime} \backsim L \widetilde{K}\left(\pi_{n}(F) \circ u^{*}, n+1\right)$, thus :

$$
\pi_{0} \operatorname{Map}_{\operatorname{sPr}(\mathcal{D}) / N G}\left(H, L \widetilde{K}\left(\pi_{n}(F), n+1\right)\right) \simeq H^{n+1}\left(H, \pi_{n}(F) \circ u^{*}\right)
$$

\subsection{Simplicial Modules Cohomology}

It is well known that for a commutative monoid $B$ in $\left(S e t, \times, \mathbb{F}_{1}\right)$, there is an equivalence

$$
s \operatorname{Pr}(\mathcal{B} B) \simeq s B-\bmod
$$

where $\mathcal{B} B$ is the category with one object with a set of endomorphisms isomorphic to $B$. We will identify these two categories in this part. Let now $A$ be a commutative monoid in sets and $B \rightarrow A$ be a morphism of commutative monoids. We are in a particular case of previous section, the category $\mathcal{D}$ is $\mathcal{B} B$ and the presheaf of groupoids $G$ is just $A$. Let $M$ be a local system on $\mathcal{B} B$, there is an isomorphism

$$
H^{n}(A, M) \simeq \pi_{0} M a p_{s B-\bmod / A}(A, L \widetilde{K}(M, n+1)) .
$$

Let $Z$ denote the abelianization functor from $B-\bmod / A$ to the category of abelian group objects in $B-\bmod / A$, denoted $A b(B-\bmod / A)$. There is an equivalence between $A b(B-\bmod / A)$ and the category of $A$ graduated $Z(B)$-modules, denoted $Z(B)-\bmod ^{A-g r a d}$. The following functor realizes this equivalence, its inverse is the forgetful functor.

$$
\Theta:(M \stackrel{f}{\longrightarrow} A) \in A b(s B-\bmod / A) \rightarrow \oplus_{m \in A} f^{-1}(m) \in Z(B)-\bmod ^{A-g r a d}
$$

This equivalence lifts to simplicial categories and it is easy to see that

$$
H^{n+1}(A, M) \simeq \pi_{0} \operatorname{Map}_{Z(B)-\bmod ^{A-\operatorname{grad}}}(Z(A), L \widetilde{K}(M, n+1))
$$

Here is the proposition that interrests us.

Proposition 2.19. Let $B \rightarrow A$ be a morphism of commutative monoids in sets. The morphism $B \rightarrow A$ is $h f$ if and only if

$\diamond Z(A)$ is homotopically finitely presented in $Z(B)-\bmod ^{A-g r a d}$.

$\diamond A$ is homotopically finitely presented for the 1-truncated model structure i.e. in the category B-Gpd.

Proof

Let us prove first the easiest part. Let $A$ be an homotopically finitely presented object in $s B-\bmod$. Let $s B-m o d \leq 1$ denotes the category $s B-\bmod$ endowed with its 1 -truncated model structure. In the adjunctions 


$$
\begin{gathered}
s B-\bmod \underset{I d}{\stackrel{I d}{\rightleftarrows}} s B-\bmod \leq 1 \\
s B-\bmod Z \underset{i}{\rightleftarrows} s Z(B)-\bmod / A \\
s B-\bmod / A Z \underset{i}{\rightleftarrows} s Z(B)-\bmod ^{A-g r a d}
\end{gathered}
$$

the left adjoint functors preserve weak equivalences and cofibrations thus the right adjoints preserve homotopically finitely presentable objects.

Let us now prove the hardest part. We start with this lemma:

Lemma 2.20. There exists $m_{0} \in \mathbb{N}$ such that for any local system $M$ and all $n \geq m_{0}$

$$
H^{n}(A, M) \simeq * .
$$

Proof

The isomorphism 1 proves that the cohomology of $A$ is isomorphic to the Ext functors of $Z(A)$ in $s Z(B)-m o d^{A-g r a d}$. Moreover, there is an equivalence of abelian categories

$$
s Z(B)-\bmod ^{A-\operatorname{grad}} \simeq C^{-}(\mathcal{B} B / A, A b)
$$

which induces by 2.18 an equivalence with the derived functors of $H^{0}$. In particular as $Z(A)$ is homotopically finitely presented, the derived functors of $H^{0}$ vanished after a set rank denoted $m_{0}$.

Remark 2.21. Two corollaries comes now. They are a consequence of this lemma and the following short exact sequence, $C \in A / s B-\bmod$

$$
\begin{gathered}
M a p_{s B-\bmod / \tau_{\leq n-1} C}\left(A, \tau_{\leq n} C\right) \longrightarrow \operatorname{Map} p_{s B-\bmod }\left(A, \tau_{\leq n} C\right) \\
\operatorname{Map}_{s B-\bmod / N G}\left(A, L \widetilde{K}\left(\pi_{n}(C), n+1\right)\right) \operatorname{Map}_{s B-\bmod }\left(A, \tau_{\leq n-1} C\right)
\end{gathered}
$$

Corollary 2.22. Let $A \stackrel{v}{\longrightarrow} C$ be in $A / s B-\bmod$. For all $i \geq 1$, for all $n \geq n_{i}=n_{0}+i+1$

$$
\begin{aligned}
\pi_{0} M a p_{s B-\bmod }\left(A, \tau_{\leq n-1} C\right) & \simeq \pi_{0} M a p_{s B-\bmod }\left(A, \tau_{\leq n} C\right) \\
\pi_{i}\left(\operatorname{Map}_{s B-\bmod }\left(A, \tau_{\leq n-1} C\right), v\right) & \simeq \pi_{i}\left(\operatorname{Map}_{s B-\bmod }\left(A, \tau_{\leq n} C\right), v\right)
\end{aligned}
$$

Proof

We first prove that the simplicial set $M a p_{s B-\bmod / \tau_{\leq n-1} C}\left(A, \tau_{\leq n} C\right)$ is not empty. There are pushout squares :

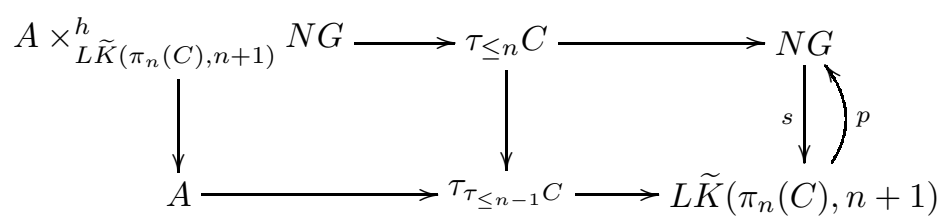

where $p \circ s=I d$. There are then equivalences

$$
M a p_{s B-\bmod / \tau_{\leq n-1} F}\left(A, \tau_{\leq n} C\right) \simeq \operatorname{Map}_{s B-\bmod / L \widetilde{K}\left(\pi_{n}(C), n+1\right)}(A, N G) \simeq M a p_{s B-\bmod / A}\left(A, A \times_{L \widetilde{K}\left(\pi_{n}(C), n+1\right)}^{h} N G\right) .
$$

Let $f$ be the morphism from $A$ to $L \widetilde{K}\left(\pi_{n}(C), n+1\right)$. There is a morphism $p \circ f: A \rightarrow N G$. As the cohomology of $A$ vanished for $n \geq n_{0}$, the elements $s \circ p \circ f$ and $f$ of the cohomology group are equals and thus

$$
p \circ f \in \pi_{0} M a p_{s B-\bmod / L \widetilde{K}\left(\pi_{n}(C), n+1\right)}(A, N G) .
$$

Then, for $i=0$, the corollary is a clear consequence of lemma 2.20 and the short exact sequence of remark 2.21 , Now, Let us study the case $i>0$. As $N G \times_{L \widetilde{K}\left(\pi_{n}(C), n+1\right)}^{h} N G \simeq L \widetilde{K}\left(\pi_{n}(C), n+1\right)$, we obtain

$$
A \times{ }_{L \widetilde{K}\left(\pi_{n}(C), n+1\right)}^{h} N G \simeq L \widetilde{K}\left(\pi_{n}(C) \circ v^{*}, n\right)
$$


Thus

$$
\pi_{i}\left(\operatorname{Map}_{s B-\bmod / \tau_{\leq n-1} C}\left(A, \tau_{\leq n} C\right), v\right) \simeq \pi_{i}\left(\left(\operatorname{Map} \operatorname{sB}_{\bmod / A}\left(A, L \widetilde{K}\left(\pi_{n}(C) \circ v^{*}, n\right)\right), q\right) \simeq H^{n-i}\left(A, \pi_{n}(C)\right)\right.
$$

where $q$ is the natural morphism from $A$ to $L \widetilde{K}\left(\pi_{n}(C) \circ v^{*}, n\right)$. We deduce then the result from lemma 2.20 and the short exact sequence of remark 2.21.

Corollary 2.23. Let $A \stackrel{v}{\longrightarrow} C$ be in $A / s B-\bmod$. The pointed tower of fibrations

$$
\left(\operatorname{Map}_{s B-\bmod }\left(A, \tau_{\leq n} C\right), v\right)
$$

converges completly in the sense of [GJ].

Proof

It can be checked with the corollary 2.21 of the complete convergence lemma of [GJ].

Corollary 2.24. For all $i \geq 0$, all $n \geq n_{i}$ and all $A \stackrel{v}{\longrightarrow} C$ in $A / s B-m o d$, there are isomorphisms

$$
\pi_{i}\left(\operatorname{Map}_{s B-\bmod }(A, C), v\right) \simeq \lim _{n \in \mathbb{N}} \pi_{i}\left(\operatorname{Map} \sin _{s-\bmod }\left(A, \tau_{\leq n} C\right), v\right) \simeq \pi_{i}\left(\operatorname{Map} p_{s B-\bmod }\left(A, \tau_{\leq n_{i}} C\right), v\right)
$$

Proof

The first isomorphism is a consequence of Milnor exact sequence ([GJ], 2.15) and the vanishing of the $\lim ^{1}$ induced by the complete convergence. The second isomorphism is a consequence of corollary 2.22

Let us now recall a well known lemma with which we will prove the last technical lemma necessary for the proof of 2.19 .

\section{Lemma 2.25. Let}

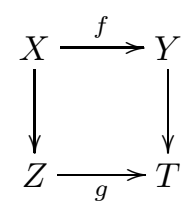

be a commutative square in sSet where $g$ is a weak equivalence. The morphism $f$ is a weak equivalence if and only if for all $z \in Z$, the homotopic fibers $X_{z}$ and $Y_{g(z)}$ are simultaneously empty and equivalent when not empty.

Here is the last technical lemma:

Lemma 2.26. Let $C \backsim$ Hocolim $_{\alpha \in \Theta}\left(C_{\alpha}\right)$ be an homotopical filtered colimit. There is a weak equivalence in sSet

$$
\operatorname{Map} \operatorname{simod}_{\bmod }(A, C) \simeq \operatorname{Hocolim} \operatorname{Map}_{s B-\bmod }\left(A, C_{\alpha}\right) .
$$

Proof

By induction on the truncation level $n$ of $C$. This is an hypothesis of 2.19 for $n=1$. Let us assume that is is true for $n-1$. Let $C$ be an $n$-truncated object in $s B-\bmod$ and $\bar{u}$ be in $\operatorname{HocolimMap} p_{s-\bmod }\left(A, \tau_{n-1} C_{\alpha}\right)$, represented by $u \in \operatorname{Map} \operatorname{simod}_{s-\bmod }\left(A, \tau_{n-1} C_{\alpha_{0}}\right)$. Let $\tilde{u}$ denote its image in $\operatorname{Map}_{s B-\bmod }(A, C)$. The filtered hocolimit along $\Theta$ is weak equivalent to the hocolimit along $\alpha_{0} / \theta$. We will use previous lemma, computing the fibers along $u$ as in the following diagram:

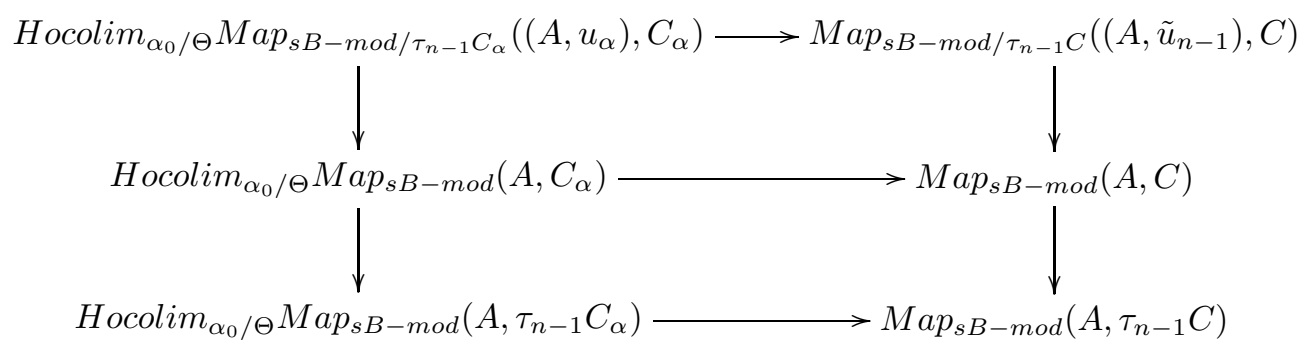

Where $u_{\alpha}: A \stackrel{u}{\longrightarrow} C_{\alpha_{0}} \longrightarrow C_{\alpha}$ and $\tilde{u}_{n-1}: A \stackrel{\tilde{u}}{\longrightarrow} C \longrightarrow \tau_{n-1} C$.

Let us show first that the fibers are simultaneously empty. The naturel morphism 


$$
\begin{gathered}
\operatorname{Hocolim}_{\alpha_{0} / \Theta} \operatorname{Map}_{s B-\bmod }\left(A, \tau_{n-1} C_{\alpha}\right) \rightarrow \operatorname{Map}_{s B-\bmod }\left(A, \tau_{n-1} C\right) \\
\bar{u} \rightarrow \tilde{u}
\end{gathered}
$$

induces the naturel morphism on cohomology groups

$$
\text { Hocolim }_{\alpha_{0} / \Theta} H^{n+1}\left(A, \pi_{n} C_{\alpha}\right) \rightarrow H^{n+1}\left(A, \pi_{n} C\right)
$$

which is a weak equivalence. Indeed, the $H^{n}$ are isomorphic to $E x t$ functors in $s Z(B)-$ mod $^{A-g r a d}$ which commute with filtered hocolimits by the first hypothesis of 2.19. The images of $\bar{u}$ and $\tilde{u}$ in the cohomology groups vanish then simultaneously, and the fibers are simultaneously empty.

Let us assume now that the fibers are unempty and prove that they are equivalent. The functors $\pi_{i}$ commute with homotopical filtered colimits, applying them on the fibers, we get the following natural morphism

$$
\operatorname{colim}_{\alpha_{0} / \Theta} \pi_{i} M a p_{s B-\bmod / \tau_{n-1} C_{\alpha}}\left(\left(A, u_{\alpha}\right), C_{\alpha}\right) \rightarrow \pi_{i} M a p_{s B-\bmod / \tau_{n-1} C}\left(\left(A, \tilde{u}_{n-1}\right), C\right)
$$

As these $\pi_{i}$ are in fact isomorphic to $H^{n-1}$, these morphisms are isomorphisms. By 2.25, this ends the proof of the lemma.

Let us now prove 2.19

Let $v: A \rightarrow C$ be in $A / s B-\bmod$ such that $C \backsim \operatorname{Hoclolim}\left(C_{\alpha}\right)$. Let us prove that the morphism

$$
\operatorname{Hocolim}\left(\operatorname{Map}_{s B-\bmod }\left(A, C_{\alpha}\right)\right) \rightarrow \operatorname{Map}_{s B-\bmod }(A, C)
$$

is a weak equivalence. Let $i$ be a positive integer, to check if the image of this morphism by $\pi_{i}$ is an isomorphism, we can just consider the case $C n$-truncated by 2.24. As the truncation commuta with homotopical filtered colimits, this is a consequence of 2.26. This ends the proof of 2.19,

\section{Examples}

\subsection{The Category $\left(\mathbb{Z}-\bmod , \otimes_{\mathbb{Z}}, \mathbb{Z}\right)$}

In classical algebraic geometry, the notion of (projective) resolution is obtained using chain complex of modules or rings. In facts, considering the correspondence of Dold-Kan this method is equivalent to taking cofibrant resolution in the simplical category ( $\mathrm{cf}[\mathrm{Q})$.

THEOREM 3.1. (Dold-Kahn correspondance)

Let $A$ be a ring. There is an equivalence of categories:

$$
s A-\bmod \simeq C h(A-\bmod ) \geq 0 \text { and } \forall i \pi_{i}(\operatorname{Map}(\mathbb{Z}, X)) \simeq H_{i}(X) .
$$

In particular, it induces a correspondence between weak equivalences and quasi-isomorphisms.

Remark 3.2. Let $A$ be a ring. Generating cofibrations of $C h(A-\bmod )^{\geq 0}$ are levelwise equal to $\{0\} \rightarrow A$ or $I d_{A}$.

Definition 3.3. Let $A$ be a rings, $M, N$ be two $A$-modules.

i. Define $\operatorname{Tor}_{*}^{A}(M, N):=H_{*}\left(M \otimes_{A}^{L} N\right)$.

ii. Define $\operatorname{Ext}_{A}^{*}(M, N):=H^{*}\left(R \underline{\operatorname{Hom}}_{A-\bmod }(M, N)\right)$.

iii. Define the projective dimension of $M$ by:

$$
\operatorname{ProjDim}_{A}(M):=\inf \left\{n \text { st } \operatorname{Ext}_{A}^{n+1}(M,-)=\{0\}\right\} .
$$

iv. Define the Tor-dimension of $M$ by:

$$
\operatorname{TorDim}_{A}(M):=\inf \left\{n \text { st } \forall X p-\text { truncated } \operatorname{Tor}_{i}^{A}(M, X)=\{0\} \forall i>n+p\right\}
$$

Remark 3.4. The functor of Dold-Kan correspondence is a strong monoidal functor, as a consequence the Tor dimension can be computed with $\pi_{i}$ instead of $H_{i}$.

Lemma 3.5. Let $X$ be in $H o(s S e t)$ and $M$ be in $s \mathbb{Z}-\bmod ($ resp $s A-\bmod$, for $A$ a ring) 
$\diamond$ The object $X$ is $n$-truncated if and only if $\operatorname{Map}(*, X) \simeq \operatorname{Map}\left(S^{i}, X\right) \forall i>n$ in $H o(s S e t)$.

$\diamond$ The object $M$ is $n$-truncated if and only if $M a p_{s \mathbb{Z}-\bmod }(\mathbb{Z}, M)$ (resp $M a p_{s A-\bmod }(A, Z)$ ) is n-truncated in $H o(s$ Set $)$.

Proof

For the first statement, by 2.25, we can consider equivalently the homotopic fibers of this morphism upon $M a p(*, X)$. The fiber of $\operatorname{Map}(*, X)$ is a point and the fiber of $\operatorname{Map}\left(S^{i}, X\right)$ is $\operatorname{Map}_{s S e t / *}\left(S^{i}, X\right)$. As $\pi_{j} M a p_{s S e t / *}\left(S^{i}, X\right) \backsim \pi_{i+j}(X)$, the equivalence is clear.

For the second statement, any object in $s \mathbb{Z}-\bmod$ is an homotopical colimit of free objects, i.e. $\forall N \in s \mathbb{Z}-\bmod$ there exists a family of sets $\left(\lambda_{i}\right)_{i \in I}$ such that $q N \backsim \operatorname{hocolim}_{I} \coprod_{\lambda_{i}} \mathbb{Z}$ in $H o(s \mathbb{Z}-\bmod )$. Assume that $M a p_{s \mathbb{Z}-m o d}(\mathbb{Z}, M)$ is $n$-truncated. $\operatorname{Map}_{s \mathbb{Z}-\bmod }(N, M) \backsim \operatorname{holim}_{I} \prod_{\lambda_{i}}\left(\operatorname{Map} p_{s \mathbb{Z}-\bmod }(\mathbb{Z}, M)\right)$, hence is an homotopical limit of $n$-truncated objects. by $i, n$-truncated objects in $s$ Set are clearly stable under homotopical limits.

Lemma 3.6. (cf [TV]) Let $u: A \rightarrow B$ be in $s \mathbb{Z}-$ mod. The morphism $u$ is flat if and only if

i. The natural morphism $\pi_{*}(A) \otimes_{\pi_{0}(A)} \pi_{0}(B) \rightarrow \pi_{0}(B)$ is an isomorphism.

ii. The morphism $\pi_{0}(u)$ is flat.

In particular, if $A$ is cofibrant and $n$-truncated, $u$ flat implies $B$ n-truncated.

Remark 3.7. [TV] Let $A \rightarrow B$ be in $\mathbb{Z}-$ alg. The morphism $A \rightarrow B$ is flat if and only if $\operatorname{Tor}_{\operatorname{Dim}}(B)=0$.

We give now the lemmas necessary to the theorem of comparison of the notions of smoothness in rings and relative smoothness.

Lemma 3.8. Let $A \rightarrow B$ be a smooth morphism of rings. There exists a pushout square

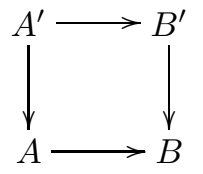

such that $A^{\prime} \rightarrow B^{\prime}$ is a smooth morphism of noetherian rings.

Proof:

This is the affine case in the corollary $17.7 .9(b)$ of [EGAIV].

Lemma 3.9. Let $A \rightarrow B$ and $A \rightarrow C$ be two morphisms in $\mathbb{Z}-$ alg. If $B$ is a perfect complex of $B \otimes_{A} B$ modules then $D:=B \otimes_{A} C$ is a perfect complex of $D \otimes_{C} D$ modules.

Proof:

Perfect complexes are clearly stable under base change. As $D \otimes_{C} D \simeq B \otimes_{A} D$, the natural morphism $D \otimes_{C} D \rightarrow D$ is a pushout of $B \otimes_{A} B \rightarrow B$ hence $D$ is a perfect complex.

Lemma 3.10. Let $A$ be a noetherian ring. Every flat A-module of finite type is projective.

Lemma 3.11. Assume that $A$ is a noetherian ring and consider $A \rightarrow B \in \mathbb{Z}-$ alg, $B$ of finite type. There is an equivalence between

i. The ring $B$ is of finite Tor-dimension on $A$.

ii. The ring $B$ is of finite projective dimension on $A$.

The part $i i \Rightarrow i$ is clear, if $B$ has a finite projective resolution $0 \rightarrow P_{n} \rightarrow \ldots \rightarrow B$, then for $i \geq n, \operatorname{Tor}^{i+1}(M,-) \backsim$ $\operatorname{Tor}^{i-n}\left(P_{n+1},-\right)$ and $P_{n+1}=0$.

Reciprocally, if $\operatorname{Tor}_{\operatorname{Dim}} \mathrm{A}<+\infty$, let $\ldots \rightarrow P_{n} \rightarrow \ldots \rightarrow B$ be a free resolution of $B$. The module $P_{n} / i m\left(P_{n+1}\right)$ has Tor dimension 0 by previous formula hence is flat by 3.7. As $A$ is noetherian and $B$ is of finite type, it is projective and we have a clear finite projective resolution. 
Lemma 3.12. Let $u: A \rightarrow B$ be in rings. Assume that $A$ is an algebraically closed field, then there is an equivalence

$\diamond$ The morphism $u$ is formally smooth in the sense of rings.

Any morphism $x: B \rightarrow A$ in rings provides $A$ with a structure of $B$-module of finite projective dimension over $B$.

Lemma 3.13. Let $u: A \rightarrow B$ be a finitely presented flat morphism in rings. The morphism $u$ is smooth if and only if for all algebraically closed field $K$ under $A, K \rightarrow K \otimes_{A} B$ is smooth.

THEOREM 3.14. A morphism $A \rightarrow B$ in $\mathbb{Z}$ - alg is smooth in the sense of rings if and only if

i. The ring $B$ is finitely presented in $A-$ alg.

ii. The morphism $A \rightarrow B$ is flat.

iii. The ring $B$ is a perfect complex of $B \otimes_{A} B$-modules.

Proof:

Let us now prove the first part of the theorem. Assume that $A \rightarrow B$ is smooth. $i$ and $i i$ are clear.

Let us prove iii. By 3.8, as iii is stable under pushout, we just have to prove it for $A$ and $B$ noetherian. Let us prove first that $B \otimes_{A} B \rightarrow B$ is of finite Tor dimension (hence of finite projective dimension by 3.11).

Let $L$ be an algebraically closed field in $A-$ alg. Set $B_{L}:=B \otimes_{A} L$. Clearly

$$
B \otimes_{B \otimes_{A} B} L \simeq B_{L} \otimes_{B_{L} \otimes_{L} B_{L}} L
$$

hence computing the Tor dimension of $B$ over $B \otimes_{A} B$ is equivalent to compute the Tor dimension of $B_{L}$ over $B_{L} \otimes_{L} B_{L}$. The morphism $L \rightarrow B_{L} \rightarrow B_{L} \otimes_{L} B_{L}$ is smooth, by composition of smooth morphisms, over an algebraically closed field. The ring $B_{L} \otimes_{L} B_{L}$ is then smooth on afield, hence regular. Now, $B_{L}$ is a module of finite type on this regular ring thus it is a perfect complex on it. In particular, it is of finite projective dimension hence of finite Tor dimension. Finally, $B$ is of finite Tor dimension hence of finite projective dimension over $B \otimes_{A} B$. As previously, $B$ of finite type over $B \otimes_{A} B$. As these rings are noetherian, $B$ is a perfect complex. Indeed, $B$ has a finite projective resolution by $\left(P_{i}\right)$. Each $P_{i}$ is of finite Tor dimension hence of finite projective dimension.

Let us prove the second part of the theorem. Let $A \rightarrow B$ be a morphism of rings verifying $i$, ii and $i i i$. Let $K$ be an algebraically closed field under $A$. We will use 3.13 and 3.12 ,

Let $x: B \rightarrow K$ be in $\mathbb{Z}-$ alg. The following commutative diagram is an homotopic pushout:

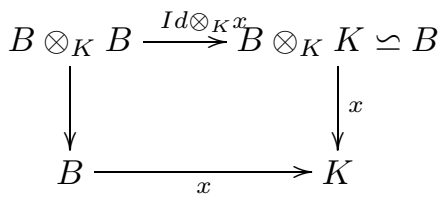

Thus $K$ has finite projective dimension in $B-\bmod$. Finally, by 3.13, $K \rightarrow B$ is smooth in the sense of rings. As it is true for any $K$, by 3.12, $A \rightarrow B$ is smooth in the sense of rings.

Here is now the comparison theorem.

THEOREM 3.15. Let $A \rightarrow B$ be a morphism of rings. It is smooth if and only if it is smooth in the sense of rings.

Proof

The two following lemmas, and remark 3.7 prove the theorem.

Lemma 3.16. [TV] Let $A \rightarrow B$ be a morphism in $\mathbb{Z}-$ alg.

i. if $A \rightarrow B$ is $h f p$, then it is finitely presented in $\mathbb{Z}-$ alg.

ii. if $A \rightarrow B$ is smooth and finitely presented, then it is $h f p$.

Lemma 3.17. reftv Let $A \rightarrow B$ be a morphism of rings. The ring $B$ is a perfect complex of $B$-modules if and only if $A \rightarrow B$ is $h f$. 


\subsection{The category Set}

The most difficult problem consists in finding examples of formally smooth morphisms. The Lemma 2.19 gives us a characterisation of these morphisms in the relative context $\mathrm{C}=$ Set.

The functor nerve and the functor "fundamental groupoid" define a Quillen equivalence between the category $s B-m o d$ endowed with its 1 -truncated model structure and the category $B-G p d$. Moreover, this last category is compactly generated and thus its filtered Hocolim can be computer as filtered colimits. Here is the formula to do this

Lemma 3.18. Let $\mathcal{J}$ be a filtered diagram and $F: \mathcal{J} \rightarrow G p d$. The colimit of $F$ consists of

$\diamond$ On objects

$$
(\operatorname{ColimF})_{0}:=\operatorname{Colim}(f g \circ F)
$$

where $f g$ is the forgetful functor from Gpd to Set.

$\diamond$ On morphisms, for $\bar{x}, \bar{y} \in \operatorname{Colim}(f g \circ F)$ represented by $x \in F(i)$ and $y \in F\left(i^{\prime}\right)$. There exists $k$ under $i$ and $i^{\prime}$ such that

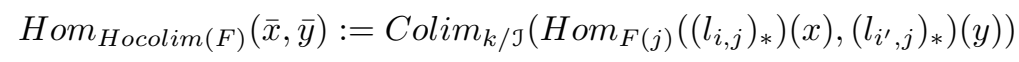

where $l_{i, j}: i \rightarrow j$ and $l_{i^{\prime}, j}: i^{\prime} \rightarrow j$.

We also need to describe the derived enriched Homs.

Lemma 3.19. Let $B$ be a monoid in Set. There is an equivalence of categories between Ho $(B-G p d)$ and the category $[B-G p d]$ whose objects are B-groupoids and morphisms are isomorphism classes of functors. In particular, for two $B$-groupoids $G$ and $G^{\prime}, \underline{R o m}_{B}^{\Delta \leq 1}\left(G, G^{\prime}\right) \simeq \underline{H o m}_{[B-g p d]}^{\Delta \leq 1}\left(G, G^{\prime}\right)$ in Ho(Gpd), where the exponent $\Delta \leq 1$ means that the Homs are enriched on groupoids.

Lemma 3.20. The commutative monoid $\mathbb{N}$ is homotopically finitely presented for the 1-truncated model structure i.e. in the category $(\mathbb{N} \times \mathbb{N})-G p d$.

Let $\mathbb{N}^{2}$ denotes $\mathbb{N} \times \mathbb{N}$. Let $F: \mathcal{J} \rightarrow G p d$ be a funnctor from a filtered diagram $\mathcal{J}$ to $G p d$. We have to prove

$$
\operatorname{Hocolim}\left(\underline{\operatorname{Hom}}_{\left[\mathbb{N}^{2}-G p d\right]}^{\Delta \leq 1}(\mathbb{N}, F(-))\right) \simeq \underline{\operatorname{Hom}}_{[\mathbb{N}-G p d]}^{\Delta \leq 1}(\mathbb{N}, \operatorname{Hocolim}(F))
$$

We let the reader verify that the following functor denoted $\varphi$ define an equivalence of groupoids.

Let $\bar{H}$ be in $\operatorname{Hocolim}\left(\underline{H o m}_{\left[\mathbb{N}^{2}-G p d\right]}^{\Delta \leq 1}(\mathbb{N}, F(-))\right)$ represented by $H \in \underline{H o m}_{\left[\mathbb{N}^{2}-g p d\right]}(\mathbb{N}, F(j))$. We define $\varphi$ on objects by

$$
\varphi: \bar{H} \rightarrow \hat{H}:=n \rightarrow \bar{H}(n)
$$

Now, by construction, any morphism $\bar{\eta}$ in $\operatorname{Hocolim}\left(\underline{\operatorname{Hom}}_{\left[\mathbb{N}^{2}\right.}^{\Delta \leq 1} G p d\right]$ $\operatorname{Hom}_{\underline{H o m}_{\left[\mathbb{N}^{2}-g p d\right]}(\mathbb{N}, F(j))}\left(G, G^{\prime}\right)$. We define $\varphi$ on morphisms by

$$
\varphi: \bar{\eta} \rightarrow \hat{\eta}:=n \rightarrow \overline{\eta_{n}}
$$

Lemma 3.21. The commutative group $\mathbb{Z}$ is homotopically finitely presented for the 1-truncated model structure i.e. in the category $(\mathbb{Z} \times \mathbb{Z})-G p d$.

Proof

This is the same proof as previous lemma, replacing $\mathbb{N}$ by $\mathbb{Z}$.

Corollary 3.22. The morphisms $\mathbb{F}_{1} \rightarrow \mathbb{N}$ and $\mathbb{F}_{1} \rightarrow \mathbb{Z}$ are smooth. In particular, the affine scheme $G l_{1, \mathbb{F}} \backsim S p e c(\mathbb{Z})$, also denoted $\mathbb{G}_{m, \mathbb{F}_{1}}$ in [TVa], is smooth.

Proof

They are clearly $h f p$ and of Tor dimension zero. Their diagonal is hf for the 1-truncated model structure, thus, we just have to check that the diagonal of their abelianisation is $h f$ in the simplicial graduated category given in 2.19 The abelianisation of $\mathbb{N}$ is $\mathbb{Z}[X]$ and the abelianisation of $\mathbb{Z}$ is $\mathbb{Z}(X)$, and the morphisms $\mathbb{Z}[X] \otimes_{\mathbb{Z}} \mathbb{Z}[X] \rightarrow \mathbb{Z}[X]$ and $\mathbb{Z}(X) \otimes_{\mathbb{Z}} \mathbb{Z}(X) \rightarrow \mathbb{Z}(X)$ are $h f$ respectively in $s\left(\mathbb{Z}[X] \otimes_{\mathbb{Z}} \mathbb{Z}[X]\right)-\operatorname{Mod}^{\mathbb{N}-\text { grad }}$ and $s\left(\mathbb{Z}(X) \otimes_{\mathbb{Z}} \mathbb{Z}(X)\right)-M o d^{\mathbb{Z}-\text { grad }}$ 
Corollary 3.23. Pour tout n, le schéma $G l_{n, \mathbb{F}_{1}}$ est lisse.

Proof

This scheme is isomorphic to $\operatorname{Spec}\left(\prod_{E_{n}} \coprod_{E_{n}} \mathbb{Z}\right)([\mathrm{TVa}])$, where $E_{n}$ is the set of integers from 1 to $n$, thus as coproducts in $\operatorname{Comm}(S e t)$ are products in $S e t$, it is isomorphic to $\operatorname{Spec}\left(\mathbb{Z}^{n^{2}}\right)$. The product in set is the tensor product, thus as a finite tensor product of finite colimits of homotopically finitely presentable object, this monoid is homotopically finitely presentable, i.e. $\mathbb{F}_{1} \rightarrow \mathbb{Z}^{n^{2}}$ is a morphism $h f p$. For the same reason, the Tor dimension is still zero. We need then to prove that a finite tensorisation of the formally smooth morphism $* \rightarrow \mathbb{Z}$ (in the relative sense 1.19 ) by itself is still formally smooth. The pushout diagram

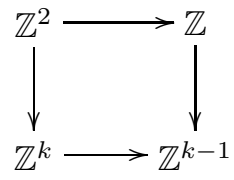

proves that $\mathbb{Z}^{k} \rightarrow \mathbb{Z}^{k-1}$ is $h f$ for any integer $k$ and by composition $\mathbb{Z}^{2 k} \rightarrow \mathbb{Z}^{k}$ is $h f$ for any integer $k$. Finaly for every $n, \mathbb{F}_{1} \rightarrow \mathbb{Z}^{n^{2}}$ is smooth, hence $G l_{n}, \mathbb{F}_{1}$ is smooth.

\subsection{Some Others examples}

If $(\mathcal{C}, \otimes, 1)$ is a symmetric monoidal category as described in the preliminaries, its associated category of simplcial objects has simplicial Homs,denoted $\underline{\mathrm{Hom}}^{\Delta}$, and there is an adjunction

$$
s \stackrel{\mathrm{Hom}^{\Delta}(1,-)}{\underset{s K_{0}}{\rightleftarrows}} s \text { Set }
$$

where $s K_{0}\left(\left(X_{n}\right)_{n \in \mathbb{N}}\right)=\left(\coprod_{X_{n}} 1\right)_{n \in \mathbb{N}}$. One verifies easily that as 1 is cofibrant, finitely presentable, and as $\underline{H o m}^{\Delta}(1,-)$ preserves weak equivalences (by construction of the model structure on $\mathcal{C}$ ), the functor $s K_{0}$ preserve homotopically finitely presentable objects. In particular, $s K_{0}$ preserves $h f$ morphisms and formally smooth morphisms. Restricting the adjunction to the categories of algebra, where weak equivalences and homotopical filtered colimits are obtained with the forgetful functor, it is also clear that $s K_{0}(u)$ preserves $h f p$ morphisms. We write then the following proposition.

Proposition 3.24. Let $u: A \rightarrow B$ be a smooth morphism in $\operatorname{Comm}(\operatorname{Set})$, then $s K_{0}(u)$ is smooth if and only if $s K_{0}(B)$ is of finite Tor dimension over $s K_{0}(A)$.

This gives particular examples. Indeed, in every context the affine line correspond to the morphism $1 \rightarrow 1[X]:=$ $\coprod_{\mathbb{N}} 1$ and the scheme $\mathbb{G}_{m}$ to the morphism $1 \rightarrow 1(X):=\coprod_{\mathbb{Z}}$. We write then the following theorem.

THEOREM 3.25. The affine line and the scheme $\mathbb{G}_{m}$ are smooth in any context where, respectively, $1[X]$ and $1(X)$ are of finite Tor dimension over 1.

This theorem can be applied in particular to the context $\mathbb{N}-\bmod$. The following lemma provides us, in this context, examples of morphisms of Tor-dimension 0 .

Lemma 3.26. Let $A \rightarrow B$ be in $\operatorname{Comm}(\mathbb{N}-\bmod )$ such that $B$ is free over $A$. The monoid $B$ has Tor-dimension 0 over $A$.

Proof :

Let $M \in A-\bmod$ be a $n$-truncated module. There exists a set $\lambda$ such that $B \backsim \coprod_{\lambda} A$. Thus $B \otimes_{A}^{L} M^{\prime} \backsim C o p r o d_{\lambda} Q M$ in $Q_{c} A-\bmod$ where $Q, Q_{c}$ are cofibrant replacement respectively in $Q_{c} A-\bmod$ and $\operatorname{Comm}(\mathbb{N}-\bmod )$. Thus as this coproduct is a product in set, we get

$$
B \otimes_{A}^{L} M^{\prime} \simeq \text { Colim }_{\lambda^{\prime} \text { finic } \lambda} \prod_{\lambda^{\prime}} Q M
$$

As functors $\pi_{i}$ commute with products in sets and filtered colimits, the Tor dimenson of $B$ over $A$ is zero.

THEOREM 3.27. Examples in $\mathbb{N}-\bmod$.

$\diamond$ The affine line in $\mathbb{N}-\bmod , \mathbb{A}_{\mathbb{N}}^{1}$, is smooth.

$\diamond$ The scheme $\mathbb{G}_{m, \mathbb{N}}$ relative to $\mathbb{N}-\bmod$ is smooth. 
We conclude with a last theorem

TheOREM 3.28. Let $\mathcal{C}$ be a relative context in the sense of $[M]$ and $A \rightarrow B$ be a Zariski open immersion in $C$ omm $(\mathcal{C})$, with $A$ cofibrant in Comm(C) and $B$ cofibrant in $A-$ alg. The morphism $A \rightarrow B$ is smooth.

Proof

A Zariski open immersion is always formally smooth, its diagonal is even an isomorphism. Thus we will need to prove that it is $h f p$ and of Tor dimension zero. First, if there exists $f \in A_{0}$, an object of the underlying set of $A$, such that $B \backsim A_{f}$, the result is clear. Indeed, $A_{f}$ is given by a filtered colimit of $A$ thus is of Tor dimension zero. Let us prove that it is $h f p$. It is clear that $A \rightarrow A[X]$ is homotopically finitely presented, then as everything is cofibrant, we can write $A_{f}$ as a finite colimit of $A[X]([\mathrm{M}])$ which is in facts a finite homotopical colimit and thus finally $A \rightarrow A_{f}$ is $h f p$.

Now if $B$ define a Zariski open object of $A$, we can write $B$ it as a cokernel of products of $A_{f}$. As functors $\pi_{i}$ commute with products, the products preserve weak equivalences and it is then clear that $A \rightarrow B$ is $h f p$. For the Tor dimension, recall that there is a finite family of functor reflecting isomorphisms $B-\bmod \rightarrow A_{f}-\bmod$. Let $M$ be a $p$-truncated $A$-module. This family sends $M \otimes_{A}^{L} B$ and its $n$-truncations, $n>p$ to the same module $Q M_{f}$ (Q is the cofibrant replacement of $A-\bmod$ ) thus clearly $M \otimes_{A}^{L} B$ is $p$ truncated and $\operatorname{TorDim}_{A}(B)=0$.

\section{References}

[A] V. Angeltveit - Enriched Reedy Categories - Proceedings of the American Mathematical Society, Vol. 136, num 7, july 2008, pp 2323-2332.

[B] K. S. Brown - Cohomology of groups - Graduate texts in mathematics, 87 - Springer-Verlag, New York-Berlin , 1982. $\mathrm{x}+308 \mathrm{pp}$.

[Bc] F. Borceux - Handbook of Categorical Algebra II - Cambridge University Press 1994 - 443 pp.

[EGAIV] A. Grothendieck - Eléments de géométrie algébrique IV - étude locale des schémas et des morphismes de schémas, partie IV - Inst. Hautes études sci. Publ. Math., num 32, 1967, 361pp.

[H] M. Hovey - Model Categories - Mathematical Surveys and Monographs, 63 - American Mathematical Society, Providence, $R I$, 1999. xii $+209 \mathrm{pp}$.

[J] J.F. Jardine - Diagrams and Torsors - K-theory 37 (2006) num 3 pp 291-309

[GJ] P. Goerss and J.F. Jardine - Simplicial Homotopy Theory. Progr. Math. 174, birkauser, 1999.

[M] F. Marty - Relative zariski Open Morphisms - Pré-publication math/

[McL] S. Mac Lane - Categories for the working mathematician - Graduate text in mathematics, 5 - Springer-Verlag, New York-Berlin , 1971. ix +262pp.

[Q] D. Quillen - On the (Co)-homology of Commutative Rings - Applications of categorical Algebra, Proc. of the Symposium in Pure Mathematics, 1968, New York - AMS, 1970.

[R] C. Rezk - Every Homotopy Theory of Simplicial Algebras Admits a Proper Model - prepublication math/0003065.

[T1] B. Toën - Champs Affines - Selecta mathematica, New Series, 12, 2006, pp 39-135

[T2] B. Toën - Derived algebraic geometry.

[TVa] B.Toën, M. Vaquié - Under Spec(Z) - pré-publication math/0509684.

[TV] B. Toën, G. Vezzozi - Homotopical Algebraic Geometry II: Geometric Stacks and Applications - Memoirs of the American Mathematical Society - Vol 193, 2008, 230pp. 